\title{
Reliability of Manual Vascular Segmentation for Retinal Fractal Dimension using Peek Retina ${ }^{\mathrm{tm}}$
}

\author{
Nur Raihan Esa, Siti Noor Hakimah Saidi, Mohd Zulfaezal Che Azemin, Nor Azwani Mohd \\ Shukri, Norsham Ahmad, Firdaus Yusof @ Alias
}

\begin{abstract}
Fractal dimension (Df) has been identified as indirect measure in quantifying the complexity of retinal vessel network which is useful for early detection of vascular changes. Reliability studies of $D f$ measurement on retinal vasculature, has been conducted on retinal images processed by using semi-automated software which only permits image with $45^{\circ}$ field of view (FOV). Smartphone-assisted fundus camera retinal image has a maximum $30^{\circ}$ FOV which warrant manual processing in measuring the Df. Retinal blood vessels need to be manually segmented to produce binary images for retinal vasculatures $D f$ measurement. Therefore, this study was conducted to determine the intragrader and intergrader reliability of manual segmentation of the retinal vasculature $D f$ measurement from retinal images taken using a smartphone-assisted fundus camera Forty-five retinal images were captured using the Portable Eye Examination Kit Retina (Peek Retina ${ }^{\mathrm{TM}}$, Peek Vision Ltd, UK). Suitable image for $D f$ analysis were selected based on gradable retinal image criteria which included; i) good image focus, ii) centered position of optic nerve head $(\mathrm{ONH})$ and iii) significant blood vessel visibility. The images were cropped 0.5 disc diameters away from disc margin and resized to $500 x 500$ pixels using GNU Image Manipulation Program Version 2.8.18 (GIMP, The GIMP Team, United States). Retinal vessels were manually traced by using layering capabilities for blood vessel segmentation. Df values of segmented blood vessels were measured by using Image $J$ (National Institutes of Health, USA) and its plugin software, FracLac Version 2.5. Intragrader and intergrader reliability was determined by comparing the $D f$ values between; two readings measured one week apart by a grader and readings from two different graders, respectively, using intraclass correlation coefficient (ICC) and Bland-Altman graphical plots. Intragrader agreement for retinal Df showed good reliability with ICC of 0.899 (95\% CI: 0.814-0.945). Bland Altman analysis indicated good agreement between $D f$ values at different grading time (mean difference $0.0050 ; 95 \%$ CI:-0.0001-0.0101). Intergrader reliability for retinal Df was high with ICC of 0.814 (95\% CI: 0.459-0.919). Bland Altman plot revealed good intergrader agreement for retinal Df between two graders with a bias value of 0.0158 (95\% CI: 0.0092-0.0223). In conclusion, manual segmentation of retinal image captured by smartphone-assisted fundus camera has good reliability $(0.75<I C C<0.9)$ for $D f$ analysis to study the morphology of retinal vasculatures.
\end{abstract}

Keywords: fractal dimension, retinal vascular, smartphone-assisted fundus camera, Peek retina, reliability

\section{INTRODUCTION}

Fractal dimension (Df) has been identified as an indirect measure of retinal vascular complexity.

\footnotetext{
Revised Manuscript Received on July 22, 2019.

Nur Raihan Esa

Siti Noor Hakimah Saidi

Mohd Zulfaezal Che Azemin

Nor Azwani Mohd Shukri

Norsham Ahmad

Firdaus Yusof @ Alias
}

A few studies on retinal Df analysis found that Df functions is a sensitive biomarker in detecting vascular structural changes [1-3].

The availability of automatic fundus camera in providing digital retinal images allows the retinal vascular complexity to be quantified. Quality of retinal image plays an important role in yielding accurate Df values [4]. Complexity analysis requires good quality retinal photographs for vascular segmentation process in producing binary images (black and white) of self-similar vessels branching patterns. Low retinal image resolution used for vascular segmentation was reported to produce an imprecise measurement of Df [4].

Segmentation of blood vessels involving manual or computer-assisted procedures has been applied in many studies. A study reported that different techniques of segmentation used to skeletonize the vascular network resulted in large variability of Df values [5]. The crucial issues in computing Df measurements are the reliability of the segmentation technique and image resolution used. Reliability analysis is important to assess the consistency of a particular method or parameter (between two measurements) to determine its applicability for the future studies [6].

In the past decades, studies on reliability of manual vascular segmentation yielded mixed results. A reliability study on the manual vascular segmentation in evaluating retinal Df demonstrated good agreement [7]. They reported that retinal Df values derived by manual segmentation techniques between two different graders showed minimal mean differences $(0.004)$ with coefficient of repeatability of \pm 0.050 . On the other hand, the Df values were shown to be less reliable between two observers of a diabetic retinopathy retinal images dataset in another study [8]. The manual segmentation might have led to under- or over segmentation of small retinal vascular structures which resulted in the inconsistent retinal Df values in the study. However, a more recent study found manual segmentation method was validated to quantify retinal Df values among hypertension and diabetic patients [9].

A few studies have been conducted to assess the intragrader and intergrader reliability of semi-automated computer programs in generating self-similar pattern of blood vessels networks for retinal vasculature Df measurements. demonstrated high reliability estimates in evaluating retinal complexity with ICC ranging from 0.93 to $0.95[10,11]$. The findings were supported by Bland-Altman analysis which revealed good intergrader agreement for retinal Df with very minimal average differences between graders. Most of the studies in 


\section{Reliability of Manual Vasculature Segmentation Method For Retinal Vascular Fractal Dimension Analysis Using Portable Eye Examination Kit Retina (Peek Retina) ${ }^{\mathrm{TM}}$}

the literature used high quality retinal images, captured by standard desktop fundus camera. Previous studies showed evidences that good retinal photography offers a sensitive and precise means of assessing the retinal vascular structural changes [12-15]. The potential of fundus images captured by smartphone-assisted fundus camera in assessing vascular complexity has yet to be explored.

Manual vascular segmentation method is the gold standard technique in tracing retinal vascular networks [7]. This method is useable to analyze any size of digital retinal images, as compared to the computer-assisted technique which only compatible to analyze image with $45^{\circ} \mathrm{FOV}$. Smaller FOV of retinal images provided by smartphone-assisted fundus cameras is suitable for manual vascular tracing in the retinal vasculatures Df measurement.

Therefore, this study was conducted to determine the intragrader and intergrader reliability of manual vasculature segmentation method using retinal images captured by a smartphone-assisted fundus camera, the Portable Eye Examination Kit Retina (Peek Retina ${ }^{\mathrm{TM}}$, Peek Vision Ltd, UK) for retinal Df measurement.

\section{METHODOLOGY}

\section{A. Study Population}

A total of 45 retinal image samples captured from a group of Malay individuals aged 17 to 50 years, with ocular refractive error (RE) less than -4.00DS, were included in this study. Retinal vessel structures are different between races with reports showed the Chinese to have larger retinal Df as compared to Malay and Indian population [16]. Thus, to avoid variables results, only Malay participants were recruited. As for RE, high myopia (>-4.00DS) was found to have smaller Df [17]. Higher RE resulted in increment of axial length which lead to alteration of retinal geometrical structures [18]. Individuals with any ocular pathologies (e.g. glaucoma, diabetic or hypertensive retinopathy), ocular media disorders (e.g. corneal opacification, cataract, or vitreous hemorrhage), systemic diseases (e.g. diabetes mellitus, hypertension and cardiovascular problems) and history of ocular trauma were excluded.

Convenience sampling technique was used for sample recruitment [19]. Participants' eligibility onto the study was determined by fulfilling the questionnaires based on the inclusion criteria. The ethical approval for the study was obtained from the International Islamic University Malaysia Research Ethics Committee (IREC; ID No: IIUM/ 504/ 14/ 11 /2 IREC 2018-226). Written consents were taken from all participants, following the tenets and declaration of Helsinki.

\section{B. Retinal Photography and Image Analysis}

The Peek Retina ${ }^{\mathrm{TM}}$ attached to a Samsung SM-G925F smartphone's eight megapixels digital camera (Samsung C\&T Corp., Seoul and Republic of Korea) was used to capture retinal images in this study. A $20^{\circ}$ to $30^{\circ} \mathrm{FOV}$ with good focus and clarity of retinal image was captured through dilated pupil. For tracing purposes, the best gradable retinal image was selected based on the following criteria:

i. Good image focus.

ii. Centered position on the optic nerve head (ONH). Any decentered images (as illustrated in Figure 1 were eliminated.

iii. Clear blood vessels visibility (as illustrated in Figure 2). Gradable retinal image was defined as the retinal image with good visibility of blood vasculature without any existence of significant opacity [11].

The image was cropped approximately 0.5 disc diameter away from the disc margin as the region of interest (ROI) and resized to 500 by 500 pixels of resolution (Figure 3 (a)) using GNU Image Manipulation Program Version 2.8.18 (GIMP, The GIMP Team, United States). GIMP Version 2.8.18 is a graphic editor software for modifying and improving digital images by editing, retouching, cropping and more specialized tasks. The usage of this software was elaborated elsewhere in the literature [11]. Optic disc center was used as a reference point for cropping purposes. The distance between reference point and outermost vertical margin was considered as a radius of the optic disc which was equivalent to 0.5 disc diameter [10].

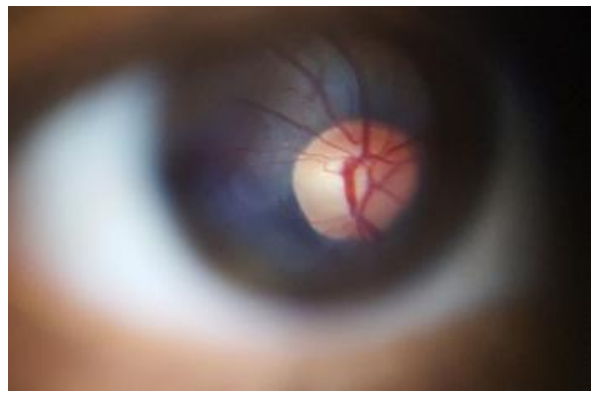

Figure 1 A decentered optic nerve head of retinal image which obstructed the appearance of retinal vasculature in the lower retinal fundus region.

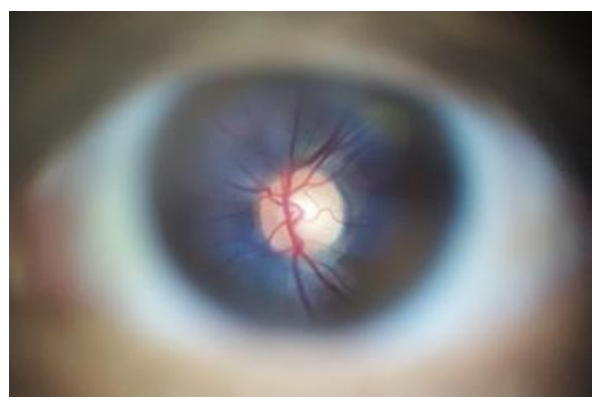

Figure 2 A good visibility of retinal blood vessels.

Manual segmentation method was adopted [9] to produce self-similar skeletonized line tracing of retinal vessels branching pattern (white color) with black background (Figure 3 (b)) using layering capabilities of the Sketchbook Pro@ drawing application (Autodesk, Inc.) and S Pen ${ }^{\circledR}$ device. Binary image of retinal vasculatures with $1028 \times 728$ size of resolution then was saved as PNG file for fractal analysis.

\section{Measurement of Df}

The Image J (National Institutes of Health, USA) with its plugins software, FracLac Version 2.5, was used to process the retinal vessel networks. It is a free, open source, scientific program developed at the National Institutes of Health and the Laboratory for Optical and Computational Instrumentation (LOCI, University of Wisconsin, USA) to process multidimensional 
image for research purposes [16]. This software is able to interpret binary digital images using box-counting algorithm to automatically calculate the Df values. The standard box count algorithm was applied to quantify the value of retinal vascular Df. Box counting manipulated the binary image by consistently computing the number of boxes containing the foreground pixels over the image ${ }^{[17]}$. The process was continued repeatedly with a different size of square boxes to obtain the slope of regression line which corresponded to the Df values [18].

The algorithm for fractal analysis was set as default using box counting options according to the standard options of box counting scan; (a) Grid position: 12; (b) Calculating grid calibers: Use default sampling sizes; (c) Minimum/maximum box size: 0 pixels $/ 45 \%$ of ROI. An average value of computed fractal dimension of retinal vasculatures over all locations was recorded.
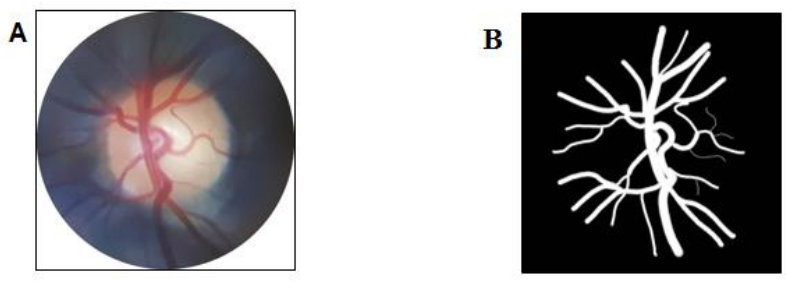

Figure 3 (a) The ROI (0.5 disc diameter) cropped retinal image; (b) A binary image of self-skeleton line tracing of retinal blood vessels branching pattern

\section{Reliability Analysis Procedures}

Intragrader reliability and intergrader reliability were defined based on a previous study [10]. Blood vessels from 45 retinal images were traced to assess the reliability of intragrader and intergrader of the retinal vessel Df measurement. Blood vessels tracing on all retinal images were conducted twice, one week apart, by one grader (Grader 1). Intragrader reliability was determined by comparing the first and second Df values. The same retinal images were traced by another grader (Grader 2). Intergrader agreement was conducted by comparing the Df values between the two graders. Df values from the first tracing of Grader 1 were taken for comparison with Grader 2 Df values. Graders were from a same background, levels of knowledge and experience.

\section{E. Statistical Analysis}

Statistical analysis procedures were performed using Statistical Package for Social Science (SPSS) software version 21. Both intragrader and intergrader reliability were assessed using intraclass correlation coefficient (ICC) and Bland Altman analysis. The ICC values were obtained by computing two-way mixed effect model and consistency type with two different measurements of 45 retinal samples. For Bland-Altman analysis, agreement between two measurements was assessed by plotting three horizontal lines; mean difference (bias value) with upper and lower limits of agreement ( $\pm 1.96 \mathrm{SD}$ of the mean differences) using scattered plots of the differences against mean of the two measurements.

\section{RESULT}

In the current study, the image samples were taken from 37
Malay female and eight Malay male with mean age of 23.09 \pm 1.49 years. The 45 retinal images were graded twice by Grader 1. The mean retinal Df values of the first and second time grading obtained were $1.4688 \pm 0.0297$ and $1.4638 \pm$ 0.0284 respectively. Meanwhile, the mean Df measurement of Grader 2 was $1.4531 \pm 0.0338$.

Intragrader agreement for retinal Df of the samples $(n=45)$ indicated a good reliability with ICC of 0.899 (95\% CI: 0.814 - 0.945) (Table I). By using Bland Altman analysis as shown in Table II, the mean difference of retinal Df for intragrader reliability analysis was 0.0050 (95\% CI: $-0.0001-0.0101)$. Figure 4 shows a good agreement between Dfs of the first time grading and the second time grading as most of the data concentrated within the limit of the agreement. The scatter plot demonstrates only three out of 45 points $(6.67 \%)$ were outside the $95 \%$ confidence intervals of agreement.

Table I Reliability estimate of intragrader and intergrader for Df measurements

$\begin{array}{cc}\text { Intraclass Correlation } & \text { p-value } \\ \text { Coefficient (ICC) } & \text { (ICC) } \\ \text { for Fractal Dimension (Df) } & \end{array}$
(95\% CI)

\begin{tabular}{lcc}
\hline Intragrader & 0.899 & \\
Grader 1 & $(0.814-0.945)$ & $<0.0001$ \\
Intergrader & & \\
Grader 1 vs & 0.814 & $<0.0001$ \\
Grader 2 & $(0.459-0.919)$ & \\
\hline
\end{tabular}

Table II Bland Altman analysis of intragrader and intergrader for Df measurements

\begin{tabular}{lcccc}
\hline & $\begin{array}{c}\text { Mean } \\
\text { Difference }\end{array}$ & SD & $\begin{array}{c}\text { 95\% CI } \\
\text { for } \\
\text { mean }\end{array}$ & $\begin{array}{c}\text { 95\% CI } \\
\text { for } \\
\text { agreement }\end{array}$ \\
\hline $\begin{array}{l}\text { Intragrader } \\
\text { Grader 1 }\end{array}$ & 0.0050 & 0.0171 & $\begin{array}{c}-0.0001, \\
+0.0101\end{array}$ & $\begin{array}{l}-0.0285, \\
+0.0385\end{array}$ \\
\hline $\begin{array}{l}\text { Intergrader } \\
\text { Grader 1 vs }\end{array}$ & 0.0158 & 0.0217 & +0.0092 & -0.0267, \\
Grader 2 & & & +0.0223 & +0.0583 \\
\hline
\end{tabular}

Intergrader agreement for retinal Df showed good reliability with ICC of 0.814 (95\% CI: $0.459-0.919$ (Table I). The bias value (mean difference) of retinal Df between Grader 1 and Grader 2 was 0.0158 (95\% CI: 0.0092 - 0.0223) (Table II). The Bland Altman plot as illustrated in Figure 5 shows good intergrader agreement for retinal Df. The graph plotted depicts only two outliers $(4.45 \%)$ lie outside the limit of agreement.

\section{DISCUSSION}

Fractal dimension analysis has been considered as a potential indicator in quantifying complexity of retinal vessel network for early detection of vascular changes[10,16]. Df measurement of retinal vessels has been evaluated by using different techniques of blood vessels segmentation. A few studies have conducted reliability analysis of retinal vasculature fractal dimension 


\section{Reliability of Manual Vasculature Segmentation Method For Retinal Vascular Fractal Dimension Analysis Using Portable Eye Examination Kit Retina (Peek Retina) ${ }^{\mathrm{TM}}$}

of semi-automated computer program in generating self-similar pattern of blood vessels networks [10,11]. In those studies, they used retinal images captured using standard fundus camera, and blood vessels were automatically traced by software. However, semi-automated method of blood vessels segmentation is only calibrated for images with $45^{\circ} \mathrm{FOV}$, the size of a typical standard desktop fundus camera captured [20]. Smaller FOV photographed by portable retinal adapter is more accessible to be traced using manual segmentation method in producing binary images for retinal branching trees vasculatures. Therefore, this study was conducted to determine the reliability of manual segmentation of the retinal vasculature Df measurement for smartphone-assisted fundus camera of PEEK retina ${ }^{\mathrm{TM}}$.

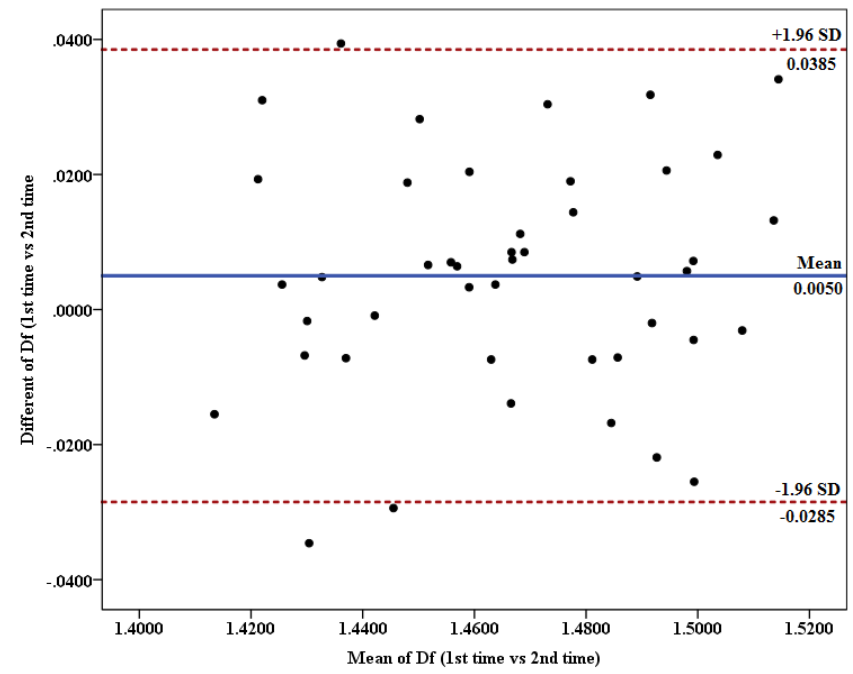

Figure 4 Limit of Agreement (LoA) of intragrader agreement for retinal Df

In this study, the mean retinal vasculatures Df were approximately similar for both Grader 1 values (1st time; $1.4688 \pm 0.0297$ and 2 nd time; $1.4638 \pm 0.0284)$ and between graders (Grader 1; $1.4688 \pm 0.0297$ and Grader 2; $1.4531 \pm$ 0.0338). The data revealed only small deviation between Df values of the manually segmented images regardless of whether the images were graded several times by a same grader, or by different graders. This result is in concert with a previous study [9] which manually segmented retinal images in patients with hypertension and diabetes . However, another study [18] reported that manual or human segmentation of retinal images was not reliable due to failure of the graders in detecting the tiny blood vessels especially in the macula region. To note, retinal image centred on the ONH were used in this study, a zone on the retina which relatively have larger vessels than the macula.

Both intragrader and intergrader of reliability were assessed using ICC and Bland Altman. ICC value of 95\% confident interval between 0 and 1 was categorized into four groups based on the ranges of reliability value of; less than 0.50 , between 0.50 and 0.75 , between 0.75 and 0.90 , and greater than 0.90 which represent poor, moderate, good, and excellent reliability respectively [19]. In the current study, the reliability estimates of retinal vessels Df of intragrader, 0.899 (95\% CI: $0.814-0.945 ; \mathrm{p}=<0.0001)$ and $0.814(95 \%$ CI: $0.459-0.919 ; \mathrm{p}=<0.0001)$ for intergrader, were found to have good agreement with ICC value lies within 0.75 and 0.90 range of reliability. These findings demonstrated that retinal images captured by the Peek Retina ${ }^{\mathrm{TM}}$ are reliable for manual segmentation of retinal blood vessels to be used for fractal analysis.

In this study, the Bland Altman findings depicted good agreement of intragrader and intergrader reliability with minimal mean differences of 0.0050 (95\% CI: -0.0001 $0.0101)$ and 0.0158 (95\% CI: $0.0092-0.0223$ ) retinal vascular Df respectively. From Bland Altman graphical plots of intra and inter agreement of retinal vascular Df, it was shown that most of the data were concentrated within the upper and lower limits of $95 \%$ confident intervals. The level of agreement is better when the range of the limits of agreement $( \pm 1.96 \mathrm{SD}$ of the mean differences) is closer to 0 with $95 \%$ of the data point lies between them [23]. These findings are in line with a study conducted by MacGillivray \& Patton, (2006) which found that manual segmentation of retinal vasculature method was reliable for fractal dimension analysis [7].

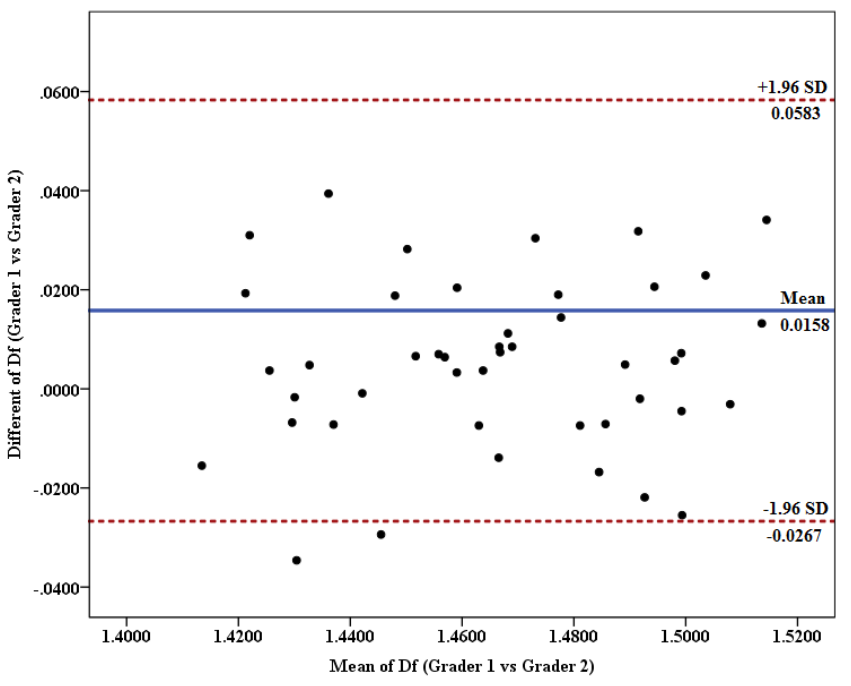

Figure 5 Limit of Agreement (LoA) of intergrader agreement for retinal Df

The widespread availability of smartphone has led to adaptability of its use in eye care. Camera adaptors such as the Peek Retina ${ }^{\mathrm{TM}}$ was developed to be used in sync with a smartphone, enables the imaging of structures inside the human eye. Smartphone-assisted fundus camera has made the fundus imaging as portable which made eye care available in remote places. The data from this study showed that images taken from this modality has good reliability in the Df analysis. It can be suggested that a smartphone-assisted fundus camera may be used to study the morphology of retinal vasculatures.

\section{ACKNOWLEDGEMENT}

This study was supported by the Research Management Centre, International Islamic University Malaysia, under Research Initiatives Grant Scheme (RIGS 16-127-0291).

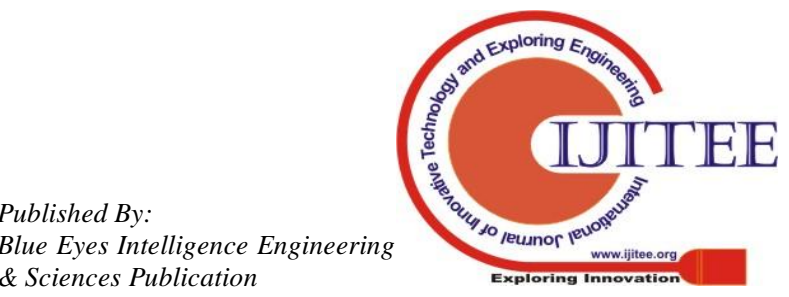




\section{REFERENCES}

1. Avakian A, Kalina R, Sage E, Rambhia A, Elliott K, Chuang E, et al. Fractal analysis of region-based vascular change in the normal and non-proliferative diabetic retina. Curr Eye Res. 2002;24(4):274-80.

2. Talu S. Fractal analysis of normal retinal vascular network. Oftalmologia. 2011;55(4):11-6.

3. Liew G, Wang JJ, Mitchell P, Wong TY. Retinal vascular imaging: a new tool in microvascular disease research. Circ Cardiovasc Imaging. 2008;1(2):156-61.

4. Huang F, Dashtbozorg B, Zhang J, Bekkers E, Abbasi-Sureshjani S, Berendschot TTJM, et al. Reliability of Using Retinal Vascular Fractal Dimension as a Biomarker in the Diabetic Retinopathy Detection. J Ophthalmol. 2016;2016.

5. Cheng SC, Huang YM. A Novel Approach to Diagnose Diabetes Based on the Fractal Characteristics of Retinal Images. IEEE Trans Inf Technol Biomed. 2003;7(3):163-70.

6. Jelinek H, Mendonça M De, Oréfice F, Garcia C, Nogueira R, Soares J, et al. Fractal Analysis Of The Normal Human Retinal Vasculature. Internet J Ophthalmol Vis Sci. 2009;8(2):1-5.

7. Bartlett JW, Frost C. Reliability, repeatability and reproducibility: analysis of measurement errors in continuous variables. Ultrasound Obs Gynecol. 2008;31:466-75.

8. MacGillivray TJ, Patton N. A reliability study of fractal analysis of the skeletonised vascular network using the "box-counting" technique. In: Annual International Conference of the IEEE Engineering in Medicine and Biology - Proceedings. IEEE; 2006. p. $4445-8$.

9. Popovic N, Radunovic M, Badnjar J, Popovic T. Fractal dimension and lacunarity analysis of retinal microvascular morphology in hypertension and diabetes. Microvasc Res. 2018;118:36-43.

10. Liew G, Wang JJ, Cheung N, Zhang YP, Hsu W, Lee ML, et al. The Retinal Vasculature as a Fractal: Methodology, Reliability, and Relationship to Blood Pressure. Ophthalmology. 2008;115(11):1951-7.

11. Ab Hamid F, Che Azemin MZ, Salam A, Aminuddin A, Mohd Daud N, Zahari I. Retinal Vasculature Fractal Dimension Measures Vessel Density. Curr Eye Res. 2016;41(6):823-31.

12. Macgillivray TJ, Trucco E, Cameron JR, Dhillon B, Houston JG, Van Beek EJR. Retinal imaging as a source of biomarkers for diagnosis , characterization and prognosis of chronic illness or long-term conditions. Br J Radiol. 2014;87(20130832):1-16.

13. Bonaldi L, Menti E, Ballerini L, Ruggeri A, Trucco E. Automatic Generation of Synthetic Retinal Fundus Images: Vascular Network Automatic Generation of Synthetic Retinal Fundus Images : Vascular Network. Procedia Comput Sci. 2016;90:54-60.

14. Huang F, Dashtbozorg B, Bart M, Romeny H. Artery / vein classification using reflection features in retina fundus images. Mach Vis Appl. 2017;1-12.

15. Kipli K, Hoque ME, Lim LT, Mahmood MH, Sahari SK, Sapawi $\mathrm{R}$, et al. A Review on the Extraction of Quantitative Retinal Microvascular Image Feature. Comput Math Methods Med. 2018;2018(4019538):1-21

16. Li X, Wong WL, Cheung CY, Cheng C, Ikram MK, Li J, et al. Racial Differences in Retinal Vessel Geometric Characteristics : A Multiethnic Study in Healthy Asians. Clin Epidemiol Res. 2013;54(5):3650-6.

17. Azemin MZ ulfaezal C, Daud NM ohamad, Ab Hamid F, Zahari I, Sapuan $\mathrm{AH}$ alim. Influence of refractive condition on retinal vasculature complexity in younger subjects. ScientificWorldJournal. 2014;2014:783525.

18. Lim LS, Cheung CY, Lin X, Mitchell P. Influence of Refractive Error and Axial Length on Retinal Vessel Geometric Characteristics. Invest Ophthalmol Vis Sci. 2011;52(2):669-978.

19. Acharya AS, Prakash A, Nigam A. Sampling: Why and How of it? INDIAN J Med Spec. 2013;4(2):330-3.

20. Abràmoff MD, Magalhães PJ, Ram SJ. Image Processing with ImageJ. Biophotonics Int. 2004;11(7):36-41.

21. Mainster MA. The fractal properties of retinal vessels: Embryological and clinical implications. Eye. 1990;4(1):235-41.

22. Losa GA. Fractals in Biology and Medicine. Second. Meyers RA editor. Switzerland: Wiley-VCH Verlag GmbH \& Co. KGaA; 2011. 6-9 p.

23. Family F, Masters BR, Platt DE. Fractal pattern formation in human retinal vessels. Phys D Nonlinear Phenom. 1989;38(1-3):98-103.

24. Fraz MM, Remagnino P, Hoppe A, Uyyanonvara B, Rudnicka AR, Owen CG, et al. Blood vessel segmentation methodologies in retinal images - A survey. Comput Methods Programs Biomed 2012;108(1):407-33.

25. Huang F, Zhang J, Bekkers EJ, Bart M, Romeny H, Dashtbozorg B, et al. Stability Analysis of Fractal Dimension in Retinal Vasculature. In: Proceedings of the Ophthalmic Medical Image Analysis Second International Workshop. 2015. p. 1-8

26. Koo TK, Li MY. A Guideline of Selecting and Reporting Intraclass Correlation Coefficients for Reliability Research. J Chiropr Med [Internet]. 2016;15(2):155-63. Available from http://dx.doi.org/10.1016/j.jcm.2016.02.012

27. Giavarina D. Understanding Bland Altman analysis Lessons in biostatistics. Biochem Medica [Internet]. 2015;25(2):141-51. Available from: http://dx.doi.org/10.11613/BM.2015.015

\section{AUTHORS PROFILE}

Nur Raihan Esa, Bachelor of Optometry (Hons.), Kulliyyah of Allied Health Sciences, International Islamic University Malaysia.

Siti Noor Hakimah Saidi, Candidate of Bachelor of Optometry (Hons), Kulliyyah of Allied Health Sciences, International Islamic University Malaysia.

Mohd Zulfaezal Che Azemin, Doctor of Philosophy, Associate Professor, Kulliyyah of Allied Health Sciences, International Islamic University Malaysia.

Nor Azwani Mohd Shukri, Doctor of Philosophy, Assistant Professor, Kulliyyah of Allied Health Sciences, International Islamic University Malaysia.

Norsham Ahmad, Doctor of Philosophy, Assistant Professor, Kulliyyah of Allied Health Sciences, International Islamic University Malaysia.

Firdaus Yusof @ Alias, Doctor of Philosophy, Assistant Professor, Kulliyyah of Allied Health Sciences, International Islamic University Malaysia. 